For Internal Distribution Only

Experimental Support and Facilities Division

Collider-Accelerator Department

BROOKHAVEN NATIONAL LABORATORY

Upton, New York 11973

ES\&F Division Technical Note \# 164

Polarization with various Time-Of-Flight cuts

Kin Yip

January 23, 2006 


\title{
Polarization with various Time-Of-Flight cuts
}

\author{
Jan. 13, 2006
}

Kin Yip

Experimental Support and Facilities Division

Collider-Accelerator Department 
This is an analysis exercise to try to see whether we can use the Time-Of-Flight cut to get more stable polarization values when the polarization target is hit by various proton intensities. The data used were those last several runs for the cold snake operation at the end of the RHIC operation in 2005 when a target scan was performed. The intensity varies as the target position varies.

\section{Section 1. Polarization under various $A_{N}$ 's}

The polarization of the proton beam is ratio of the left-right asymmetry (of the number of recoiled carbon events) over the analyzing power $A_{N}$. In the calculation, the weighted average of $\mathrm{A}_{\mathrm{N}}$ is used:

$$
\overline{A_{N}}=\Sigma\left[\mathrm{A}_{\mathrm{N}} \mathrm{I} \mathrm{N}(\mathrm{i})\right] / \Sigma \mathrm{N}(\mathrm{i})
$$

(Equation 1)

First of all, I have tried to get a set of $A_{N}$ 's that are binned evenly in TOF instead of the nominally used $-t$ bins. At the time of analysis, Larry Trueman happened to give out (internally) a new set of $A_{N}$ 's based on the pC data at $100 \mathrm{GeV}$ normalized with the JET from the 2004 run. For convenience, I have just asked him to generate the same set of $\mathrm{A}_{\mathrm{N}}$ 's but in evenly distributed TOF bins.

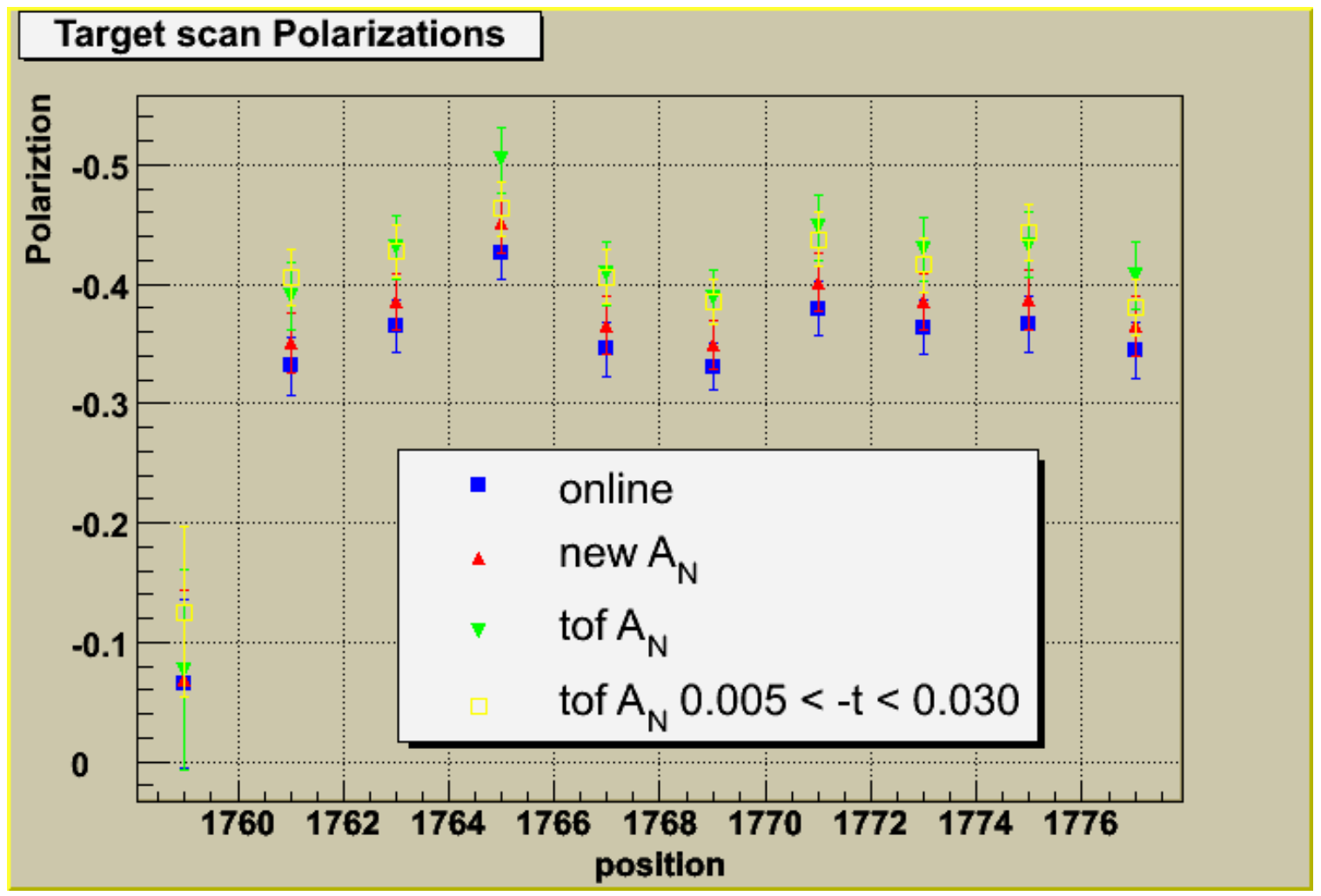

Figure 1: Various polarizations corresponding to various sets of $A_{N}$ and various cuts.

Figure 1 shows the polarizations when various sets of $A_{N}$ 's and cuts are used. The blue squares ("online") are the online values which are calculated with the official 
set of $A_{N}$ 's that have been used. The red triangles ("new $A_{N}$ ") correspond to the polarizations when Larry Trueman's new set of $A_{N}$ 's are used which have the same $-t$ binnings as the official set of $\mathrm{A}_{\mathrm{N}}$ 's. The green inverted triangles ("TOF $\mathrm{A}_{\mathrm{N}}$ ") are the polarization values if I use the same Larry Trueman's new set of $A_{N}$ 's but in equally divided TOF binnings. The allowed $-t$ range in the above cases is:

$$
0.009<-\mathbf{t}<0.022
$$

(Equation 2)

For the last set of yellow squares ("TOF $\mathrm{A}_{\mathrm{N}} 0.005<-\mathrm{t}<0.030$ ") in Figure 1, the following wider range of $-t$ is used to calculate the polarizations:

$$
0.005<-\mathbf{t}<\mathbf{0 . 0 3 0}
$$

(Equation 3)

Figure 2 shows the ratio of the polarizations between the set of ("TOF $\mathrm{A}_{\mathrm{N}}$ ") and the set of ("new $A_{N}$ "). The ratio is more or less a constant over all the target positions.

Figure 2: The ratio of polarizations between the set of points of green inverted trianges ("TOF $A_{N}$ ") and the set of points of red triangles ("new $\left.A_{N} "\right)$ in Figure 1.

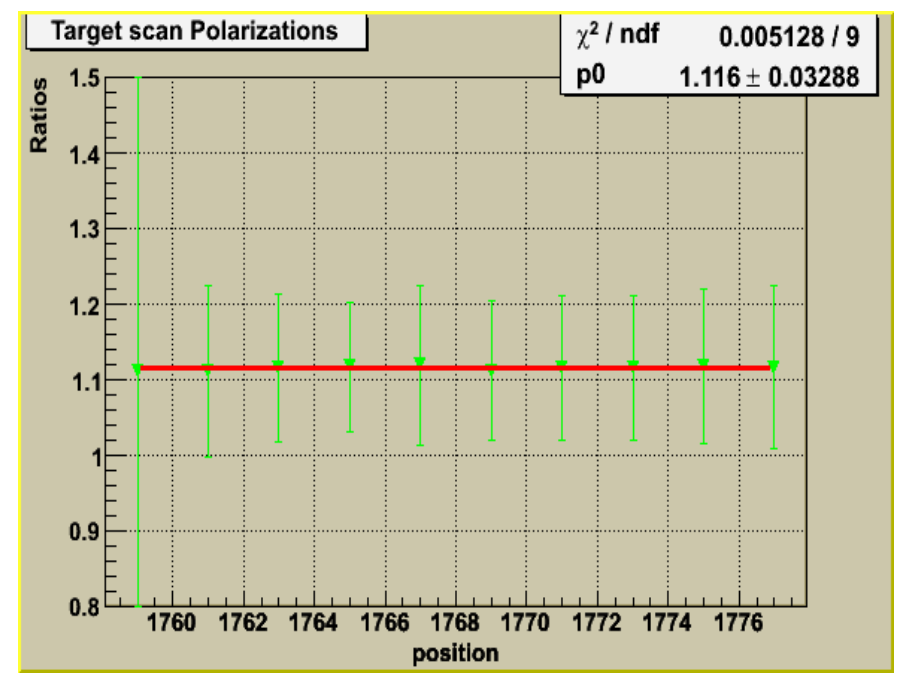

Figure 3 shows the typical banana distribution (taken from the data) and one can see where the significance of the cuts on the $-t$. 


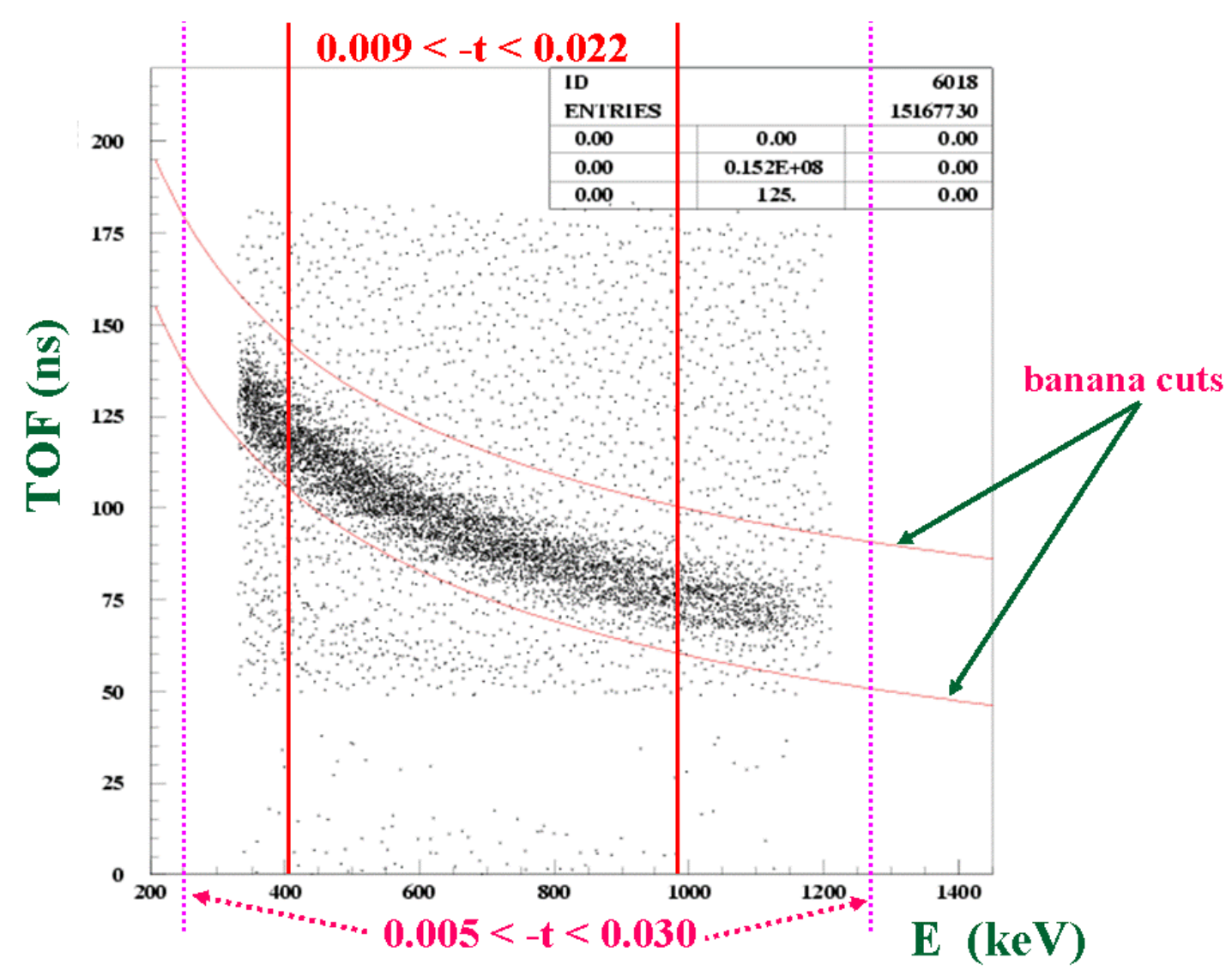

Figure 3: The banana curve (TOF versus Energy).

\section{Section 2. The effect of Varying TOF cuts}

Starting with the new set of "TOF $A_{N}$ " with TOF bins as in Section Section 1, the polarizations are recalculated with various TOF cuts. The results are shown in Figure 4. The cut variations include taking away the banana cuts and putting an additional cut of TOF $>90$ ns with and without the presence of banana cuts. The banana cuts are clearly indicatetd by the two red curves in Figure 3. One can see that various sets of polarizations move up and down more or less together at all the target positions. The polarization profile versus target positions remains basically unchanged. This is the case even with slightly harder cut such as TOF $>100 \mathrm{~ns}$ or even TOF $>110 \mathrm{~ns}$. 


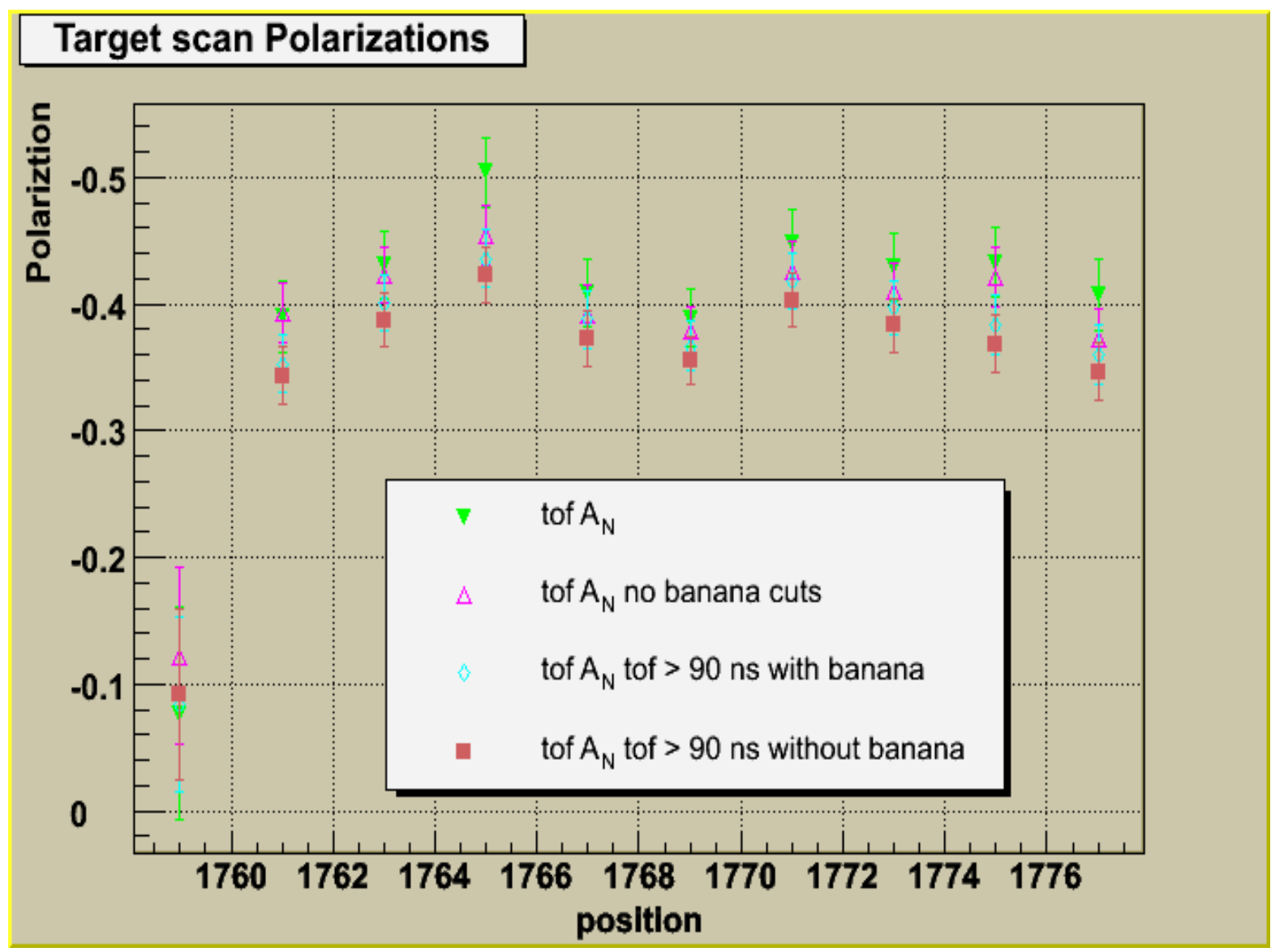

Figure 4: Variation of the polarizations under various cuts.

One thing to note here is that for the set of polarizations calculated without the banana cuts, we have almost completely got rid of the kinetic energy cuts (only the very loose $0.005<-\mathrm{t}<0.030$ cut exists). Here, only the TOF $>90$ ns has been used.

Another set of calculations with more restricted (and actually too restricted) cuts of TOF > 120 ns have also been done to see how the polarizations would be affected. This is shown in Figure 5. Here, there is some sign of change of profile, especially in the middle of the target positions. But within errors, one may still argue thing each set is flat except the lowest positions. 


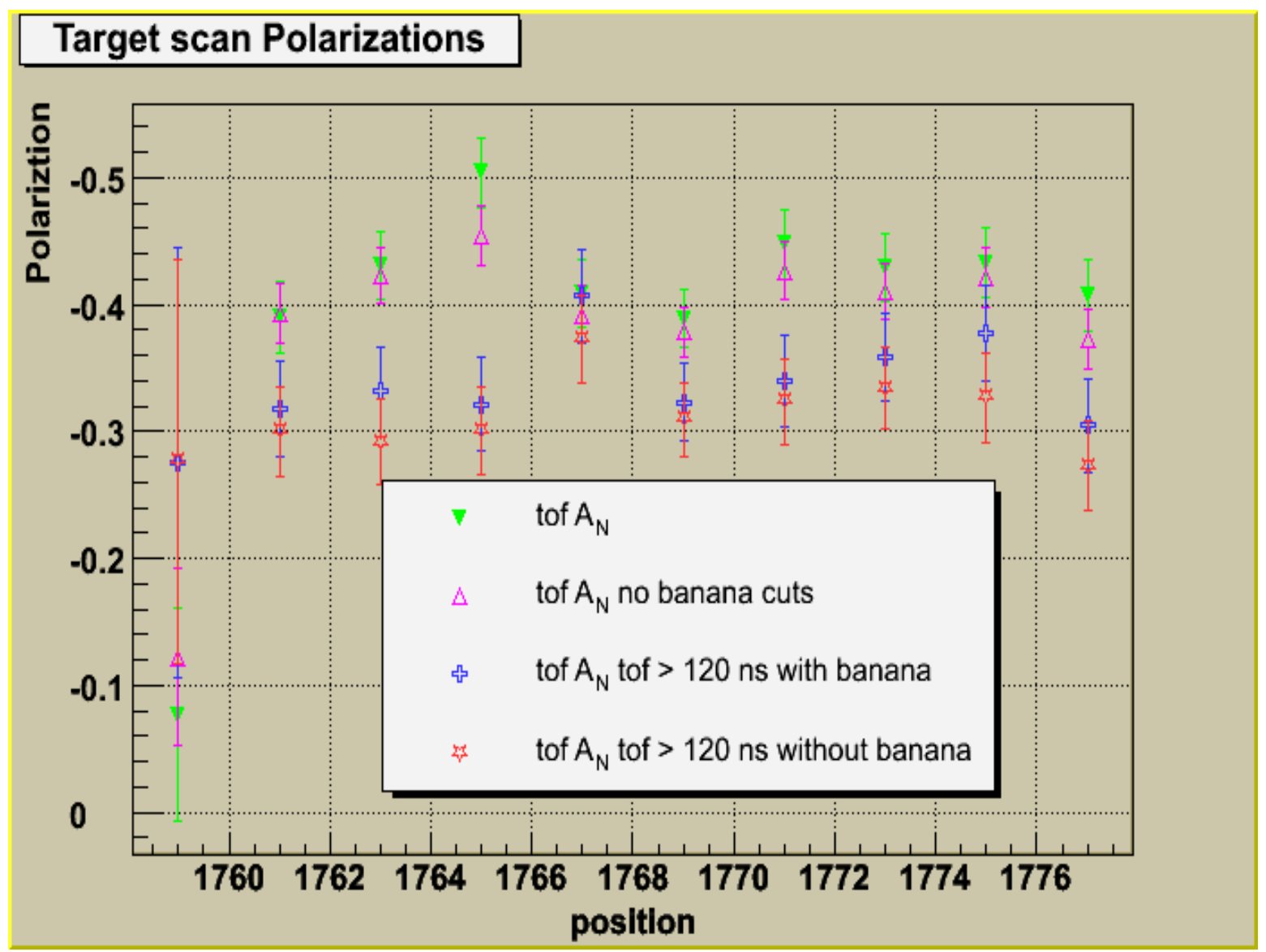

Figure 5: Variation of the polarizations similar to those in Figure 4 except this time TOF > $120 \mathrm{~ns}$ is used.

Moreover, if one looks at the banana distribution in Figure 3, most statistics are eliminated and thus the resulting plot of polarizations probably may not be the most meaningful to scrutinize upon. Furthermore, if there is a real change of profile in the middle, various TOF cuts should show consistent results. This is the not case here.

Figure 6 shows the distribution of $A_{N}$ 's versus the target positions that have been used in the calculation of polarizations as shown in Figure 5. This shows that both asymmetries and $\mathrm{A}_{\mathrm{N}}$ 's at various target positions change with the TOF and banana cuts. 


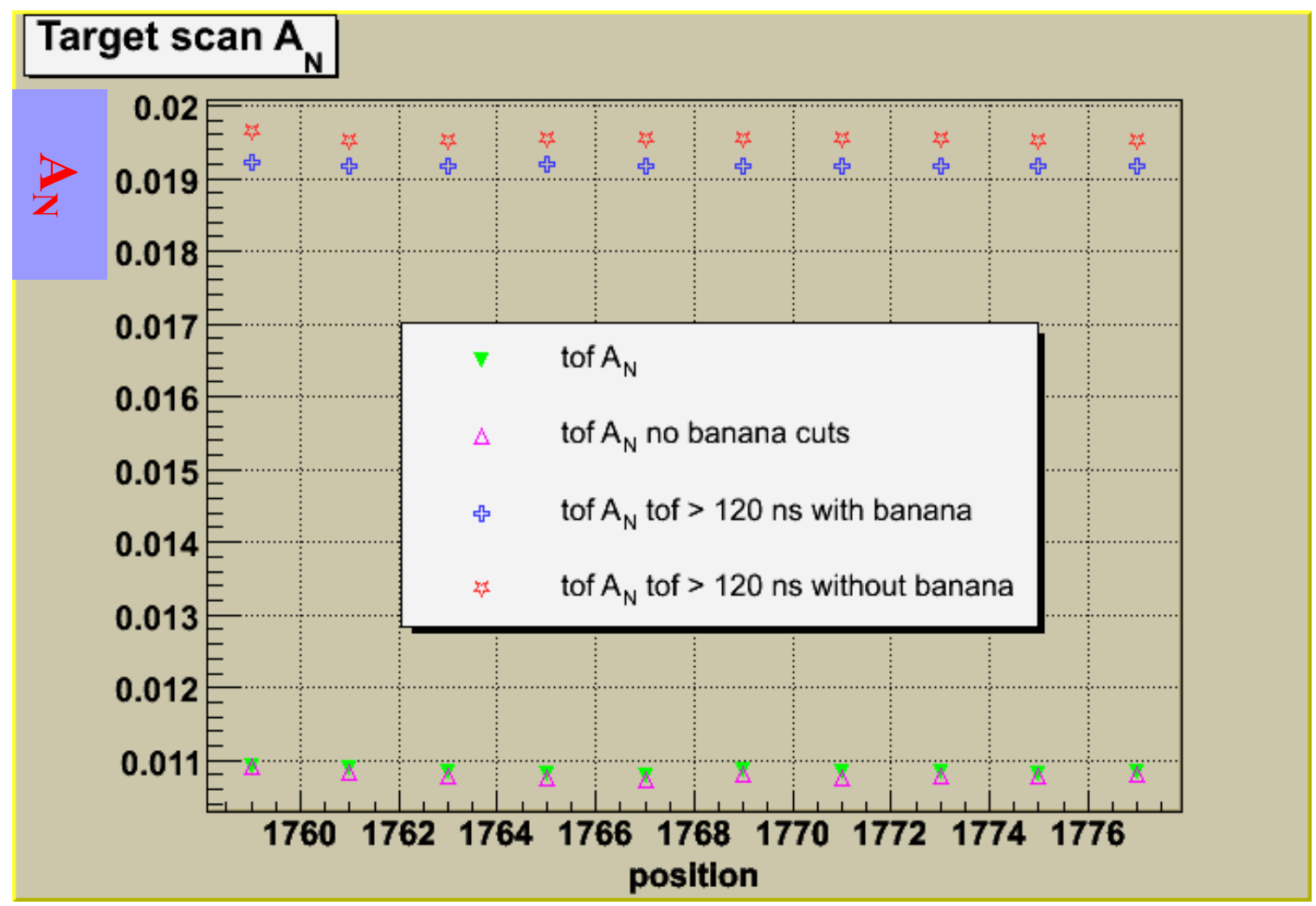

Figure 6: The distribution of $A_{N}$ 's at each target position that used in the calculations of polarizations resulting in Figure 5.

Finally, we show the distributions of TOF (ns) at two narrow bands of kinetic energy of the recoiled carbons in Figure 7. The shapes of the distribution for these two equally wide energy bands are similar but there are signicantly more concentrations of events at the lower energy band. 


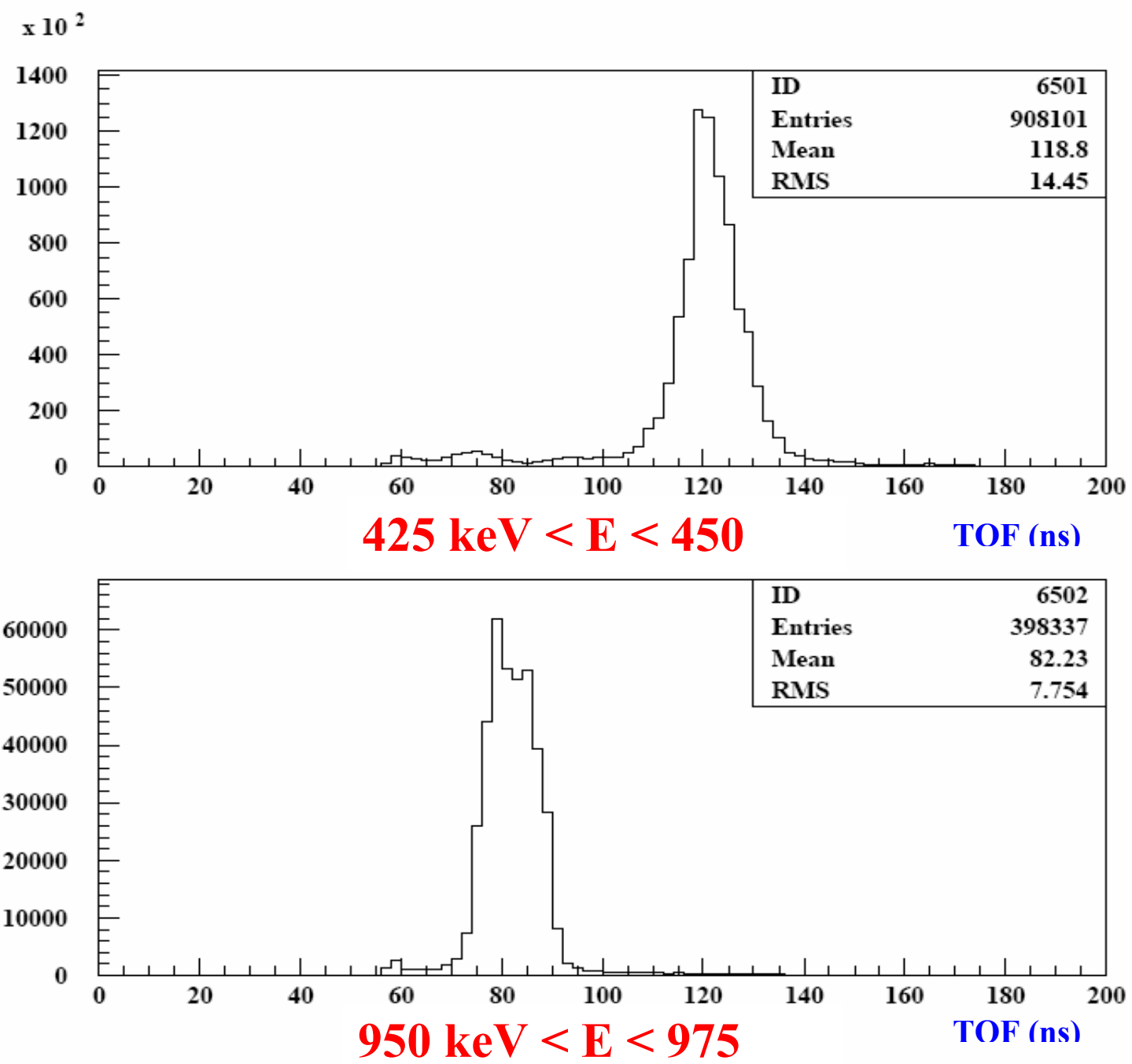

Figure 7: Distributions of TOF (ns) at two narrow bands of kinetic energy of the recoiled carbons.

\section{Section 3. Conclusion}

The idea of using TOF, especially alone, is appealing because this may get rid of the necessity to do energy and dead layer calibration frequently. When the energies are low, the energy preicisions are poor compared to those at high energies. Nevertheless, when the energies are the lowest, the TOF's are the largest and therefore have the highest precision.

This exercise was partially initiated because the accelerator physicists do not understand why the polarizations dip in the middle of the target positions when the intensities are the highest. It was hoped that using TOF cuts alone, instead of the kinetic energy cuts, may have a flat distribution. Unfortunately, the results shown in this note do not really show that using TOF would significantly change the profile of the polarizations. 
Furthermore, from past experiences, the asymmetries (and therefore the polarization values) at low energy tend to be very unstable. 